\title{
Ciencia, Tecnología y Derecho Ambiental
}

\section{Vito Leonardo Verna Coronado}

Abogado por la Pontificia Universidad Católica del Perú. Máster en Economía y Gestión Ambiental por la Universidad Comercial Luigi Bocconi. Profesor de posgrado en la Pontificia Universidad Católica del Perú. Profesor de posgrado en la Universidad Peruana de Ciencias Aplicadas. Profesor de posgrado en la Universidad ESAN.

SUMARIO:

I. Introducción.

II. Características del Derecho Ambiental.

1. Enfoque, diseño y acción sistemática.

2. Preventivo y precautorio.

3. Renovador en lo económico.

4. Sustrato metajurídico.

5. Multidisciplinario.

6. Vinculado a resultados —riesgos, crisis-.

III. Cierre de la Primera Parte.

IV. Ciencia, Tecnología y Riesgos Ambientales.

1. Caso 1.

2. Caso 2.

V. A manera de cierre. 


\title{
RESUMEN:
}

El artículo pretende ser el inicio de la exploración de las dinámicas que se producen entre la ciencia, la tecnología y el Derecho Ambiental, partiendo de la explicación de las características del Derecho Ambiental y confrontándolas con una visión de ciencia y tecnología que quizás sea algo anacrónica a la luz de lo que hoy la tecnología implica para la especie humana. Es por ello solo una invitación a investigar.

Palabras clave: ciencia, tecnología, Derecho Ambiental, sistémica, sistemas, riesgo ambiental, prevención, internalización de costos ambientales.

\begin{abstract}
:
The article intends to be the beginning of the exploration of the dynamics that take place between science, technology and Environmental Law, starting from the explanation of the characteristics of Environmental Law and confronting them with a vision of science and technology that is perhaps somewhat anachronistic in light of what technology implies for the human species nowadays. It is therefore only an invitation to investigate.

Keywords: science, technology, Environmental Law, systemic, systems, environmental risk, prevention, internalization of environmental costs.
\end{abstract}

\section{INTRODUCCIÓN}

Este artículo tiene como propósito invitar al lector a reflexionar e investigar en torno a la forma en que el Derecho Ambiental se ve influenciado en su devenir por los descubrimientos científicos y el desarrollo tecnológico.

El Derecho Ambiental es quizás una de las ramas del sistema jurídico más sensibles a lo que acontece en las ciencias naturales y planetarias. Asimismo, la sistémica - que constituye un método científico - aporta al Derecho Ambiental una herramienta fundamental de análisis y entendimiento de la realidad, además de, en mi opinión, un principio de formulación e interpretación de normas, construcción de estructuras regulatorias interrelacionadas e incluso principios ambientales derivados'.

Algunas de las conclusiones, predicciones y recomendaciones provenientes de las ciencias y tecnología ingresarán y probablemente reformarán los objetivos e instrumentos del Dere- cho Ambiental, sin embargo, antes se produce un proceso complejo donde se confronta: ciencia, incertidumbre, política, ética, bienestar humano y derecho - en su acepción de sistemática jurídica-.

La tecnología se encuentra relacionada directamente con el surgimiento y ratio del Derecho Ambiental. Se suele repetir que la norma ambiental nace precisamente de la necesidad de regular y restringir el poder que la tecnología concedió a la civilización para transformar el planeta que la cobija.

De acuerdo con el Diccionario de la Real Academia Española², la primera acepción de la palabra tecnología alude al: "Conjunto de teorías y de técnicas que permiten el aprovechamiento práctico del conocimiento científico."

La innovación tecnológica es "libre", sin embargo, fluye por causes definidos, los cuales son esculpidos constantemente por la acción de una serie de fuerzas, entre las que podemos citar

1. Puede revisarse el principio de indivisibilidad, de clara inspiración sistémica, el cual se encuentra reconocido en el artículo 3 del Reglamento de la Ley del Sistema Nacional de Evaluación e Impacto Ambiental, aprobado por Decreto Supremo Nº19-2009-MINAM y en la Guía del Servicio Nacional de Evaluación y Certificación Ambiental para las Inversiones Sostenibles - SENACE, "Lineamientos para la aplicación del Principio de Indivisibilidad en la evaluación del impacto ambiental a cargo del Senace y sus Anexos", aprobada por Resolución Jefatural N 008-2018-SENACE/JEF.

2. Cfr.: https://dle.rae.es/tecnolog\%C3\%ADa?m=form. 
a la económica, la política o la ética, las cuales suelen presentarse mezcladas en diferentes proporciones e influidas por las ideas provenientes de las teorías del desarrollo sostenible. Así, podemos concluir que la evolución tecnológica sigue una pauta, que en cualquiera de los casos es dictada por intereses, necesidades o valoraciones humanas.

La tecnología y la ciencia se encuentran muy relacionadas, sin embargo, la diferencia más saltante entre ellas radica en que mientras la tecnología evoluciona para el hombre, la ciencia evoluciona por el hombre. Dicho en otras palabras, la tecnología sirve al hombre y es él quien le otorga dirección y sentido, mientras que la ciencia es esencialmente libre, le incomodan las agendas y programas, responde únicamente a la inquietud insaciable de nuestra especie por conocer más y mejor de todo. Somos seres que vivimos con una curiosidad muy curiosa.

En las páginas que siguen se citarán algunas características del Derecho Ambiental, las cuales hemos seleccionado por ser particularmente sensibles a los acontecimientos de la ciencia y la tecnología, luego expondremos un par de casos, que en mi opinión resultan interesantes debido a que muestran la forma en que la ciencia influye o puede influir en las normas del Derecho Ambiental.

\section{CARACTERÍSTICAS DEL DERECHO AM- BIENTAL}

\section{Enfoque, diseño y acción sistémica.}

El Derecho Ambiental presenta características particulares que le confieren singularidad e identidad dentro del sistema jurídico. Una primera característica es el enfoque sistémico, abstracción que precisamente produce la visión integral de los fenómenos que constituyen su ámbito material: el juego de interrelaciones entre las conductas humanas y el sistema ambiental —compuestos por elementos bióticos y abióticos - que sustentan la vida en todas sus formas en nuestro planeta.

Esta aproximación a la realidad, característica del Derecho Ambiental, la encontramos también en el Informe "Nuestro futuro común" de las Naciones Unidas, conocido también como el Informe Bruntland, del cual citamos el siguiente extracto:

"Hasta hace poco, el planeta era un vasto mundo donde las actividades humanas y sus efectos se distribuían en netos compartimentos dentro de las naciones, los sectores energía, agricultura, comercio y amplias esferas de interés medio ambiente, economía, problemas sociales. Estos compartimentos han comenzado a desaparecer. Esto vale en Particular de las varias .crisis. mundiales que han sido motivo de la preocupación del público, en especial en el decenio pasado. No se trata de crisis separadas: crisis del medio ambiente, crisis del desarrollo, crisis de la energía. Son todas una sola crisis." ${ }^{13}$-El subrayado es nuestro-.

La sistémica nos releva una nueva forma de mirar y entender la realidad, la cual es opuesta a la simplificación o reducción que la ciencia utiliza para explicar los misterios de la física, por ejemplo. Se trata de mirar el todo y las partes a la vez, abordando la complejidad no como problema sino como revelación.

El profesor Garciandía define el sistema como: "Un conjunto de elementos, acciones o individuos que conforman una unidad global como consecuencia de la organización que surge de las interrelaciones regulares, estables, constantes y persistentes entre los mismos" ${ }^{4}$.

3. Organización de las Naciones Unidas, Informe Brundtland (Nueva York, 1987), 13.

4. José Garciandía, Pensar sistémico: una introducción al pensamiento sistémico (Bogotá: Editorial Pontificia Universidad Javeriana, 2011), 107. 
La unidad global reconocible a partir de las interrelaciones organizadas de los elementos que la conforman produce uno o varios fenómenos nuevos, que podemos llamar "propiedades" o "cualidades" las cuales reciben la denominación genérica de "emergencias", término que hace una alusión metafórica a la aparente acción de surgimiento de esta propiedad desde y por el sistema.

En este orden de ideas, la visión sistémica nos permite concebir la vida - en su maravillosa diversidad- como una emergencia, la cual, por lo tanto, solo se explica a partir del mantenimiento de la interrelación organizada que como citamos antes es: la interrelación regular, estable, constante y persistente de los componentes constituyentes del sistema ambiental.

En mi opinión, la vida, en su cambiante devenir, constituye la emergencia última y definitiva que el Derecho Ambiental busca preservar, ello implica aceptar y mantener la dinámica propia de la vida, dominada por la extinción y evolución de las especies y aligerar la impronta antropocéntrica que domina el Derecho.

Otro aporte de la teoría de sistemas a la filosofía ambiental lo representa la tolerancia a la paradoja que encierra la propia concepción de sistemas, la cual nos transporta a pensar simultáneamente en el todo como unidad organizada y al mismo tiempo en las partes que lo conforman, sin que ello suponga una contradicción irresoluble.

En palabras de Garciandía: "Las referencias al sistema son de carácter impreciso dado que hablamos del todo y de partes, aspectos ambos que parecieran contradecirse o al menos genera cierta visión paradójica del concepto sistema. ${ }^{5 "}$

La perspectiva sistémica determina no solo los procesos de aprehensión dinámica de la realidad objeto de regulación, sino además los remedios o instrumentos que el sistema jurídico ambiental usa para atender los temas ambientales. Estos instrumentos tienden cada vez más a nutrirse del enfoque sistémico —-sistémico endógeno-y a interrelacionarse con otros instrumentos, ya sean ambientales o no, aprovechando y favoreciendo la aparición de sinergias —sistémico exógeno-.

Problemas ambientales como la calidad del aire respirable son abordados a través de múltiples instrumentos que buscan resolverlos, a saber: normas técnicas sobre combustibles, estándares de emisiones, derechos transables de emisiones, fiscalización ambiental, incentivos o desincentivos fiscales, ordenamiento territorial, sistemas masivos de transporte, promoción de sistemas alternativos de transporte y un largo etc., concebidos aisladamente, pero al mismo tiempo diseñados para actuar sinérgicamente en beneficio de los objetivos ambientales de mejora de la calidad del aire.

El Derecho Ambiental se caracteriza por establecer nuevas interrelaciones entre instrumentos, jurídicos o no, en beneficio del ambiente, no solo crea nuevos instrumentos ambientales de gestión, quizás lo más relevante es que el Derecho Ambiental impone a los instrumentos no ambientales existentes formas nuevas de acción e interacción basados en objetivos jurídicos que tienen que ver con el disfrute de los derechos ambientales.

Como vimos en la lista de instrumentos citada arriba, las normas técnicas, por citar un ejemplo, existen desde antes de la aparición del Derecho Ambiental, sin embargo, hoy sirven de una forma muy relevante al cumplimiento de los propósitos ambientales. Muestra de ello es que hoy las normas ambientales consideran, con cada vez mayor frecuencia, a la actividad de normalización como pieza clave en la mejora de la calidad ambiental.

Un claro ejemplo de ello lo podemos encontrar en el artículo en la Segunda Disposición Com- 
plementaria y Transitoria del Decreto Legislativo 1278 , que aprueba la Ley de Gestión Integral de Residuos Sólidos, que subraya la importancia de la actividad de normalización para la adecuada gestión de los residuos sólidos. El artículo 31 del Decreto Supremo № 009-2019-MINAM, que aprueba el Régimen Especial de Gestión y Manejo de Residuos de Aparatos Eléctricos y Electrónicos, señala que para el manejo adecuado de estos residuos se debe considerar el cumplimiento de las Normas Técnicas Peruanas, es decir, las NTP 900.064:2012 y NTP 900.065:2012; o en el recientemente aprobado régimen que regula el plástico de un solo uso y los recipientes o envases descartables - Ley 30884 y su reglamento, aprobado por Decreto Supremo $\mathrm{N}^{\circ}$ 006-2019-MINAM-.

La visión sistémica del problema ambiental determina un funcionamiento en consonancia de la institucionalidad ambiental, la cual por su naturaleza y objetivos debiera ser integradora de las demás instituciones, colaborativa, de acción transectorial, y con un énfasis muy pronunciado en la coordinación interinstitucional y en la acción social, promoviendo la acción individual y colectiva en beneficio del ambiente.

La sistémica es al mismo tiempo la forma de aprehender el fenómeno ambiental y concebir su solución, por ello constituye parte esencial del Derecho, la acción y la institucionalidad ambiental.

La tecnología tiene la facultad de optimizar y multiplicar la acción sistémica que constituye la receta principal del Derecho Ambiental. Muchos de los instrumentos de gestión ambiental requieren de la tecnología para funcionar, de la misma forma, la acción ambiental sistémica sería imposible sin la existencia de la tecnología.

El monitoreo del bosque amazónico exige la construcción de un sistema de vigilancia ${ }^{6} \mathrm{com}$ puesto por una red de sensores ubicados en satélites, drones y radares, que tienen como objetivo monitorear no solo la cobertura del bosque primario y parámetros meteorológicos críticos para su supervivencia, tales como la temperatura del aire y la humedad, sino también nos permite ver la operatividad de actividades ilegales que lo depredan, tales como la minería y tala ilegal, la agricultura migratoria o el narcotráfico, así como las miserias humanas que las acompañan —-trabajo infantil, trata de personas y trabajo forzado-.

La información proveniente del sistema de vigilancia, que es mucha, compleja y muy rica, substancia la construcción de un tipo de regulación ambiental distinta, más holística, integradora y comprehensiva, es decir sistémica.

\section{Preventivo y precautorio.}

Otras características del Derecho Ambiental, que la doctrina especializada reconoce pacíficamente es que sus normas tienen una obsesión preventiva, la cual busca incorporar en las actividades reguladas el control de las amenazas y vulnerabilidades ambientales -riesgos ambientales-.

Mucha de la regulación ambiental encuentra su justificación —utilidad- en la prevención o mitigación de riesgos que afecten la dinámica y funcionalidad de los ecosistemas y el bienestar humano. El Derecho Ambiental impulsa un actuar que privilegia el ahora, el hoy, con la finalidad de lograr un futuro mejor, por ello de alguna forma, el Derecho Ambiental sirve como conexión entre el pronóstico del futuro -que constituye su motor-y el presente.

La representación del futuro posible o probable implica que penetremos en el campo de lo desconocido, lo eventual, lo incierto, y que acudamos a la ciencia y tecnología para acotar la incertidumbre, para que podamos ver más y mejor.

6. En el Perú se cuenta con el Sistema de Vigilancia Amazónica y Nacional —SIVAN—, que es administrado por el Centro de Vigilancia Amazónica y Nacional —CEVAN_- el cual depende de la Fuerza Aérea del Perú-FAP. Para más información consultar: https://cevan.fap.mil.pe/. 
La ciencia y la tecnología le permiten al Derecho Ambiental identificar los riesgos, calibrar su magnitud y relevancia, realizar estimaciones, formular modelos predictivos, todo lo cual sirve al objetivo primordial del Derecho Ambiental, el cual es conjurar las amenazas para la vida. No obstante, la incertidumbre persistirá.

La obstinada persistencia de la incertidumbre planteó una cuestión al jurista, ipuede el Derecho Ambiental admitir restricciones a conductas humanas cuando no existe certeza sobre los efectos negativos que estas conductas podrían producir en el ambiente? La reflexión a esta cuestión es la que fundamenta el principio precautorio, el cual admite la acción restrictiva ambiental bajo ciertas condiciones de incertidumbre.

\section{Renovador en lo económico.}

La economía como ciencia social se concebía como un sistema cerrado cuyos elementos eran el mercado, los productores y consumidores, omitiendo considerar, durante siglos, que el sistema económico era en realidad abierto y que tenía claros vínculos con el ambiente.

La economía ambiental pone de relieve la importancia que tienen los servicios ambientales como soporte irremplazable de la actividad económica; la provisión de materia - recursos renovables y no renovables - y la recepción y dispersión de desechos de la producción y el consumo, además de los sistemas de soporte de la vida, constituyen los fundamentos ambientales iniciales e irremplazables de toda dinámica económica.

Al haberse ignorado esta realidad durante gran parte de la historia de la economía y, como resultado de ello, haberse considerado los servicios ambientales como bienes públicos infinitos, hoy enfrentamos serios problemas derivados de la sobreexplotación de los recursos naturales y la saturación de los sistemas naturales de recepción y dispersión de desechos, todo lo cual está ejerciendo una enorme presión sobre la capacidad del ecosistema para continuar prestando soporte a las actividades humanas y por su puesto a la vida en su conjunto.

Racionalizar la explotación de recursos y la dis- posición de desechos hasta alcanzar niveles compatibles con la estabilidad ecosistémica implica una transformación de nuestras dinámicas tradicionales de explotación, transformación, consumo y emisión, vale decir, una evolución de nuestra economía, la cual es ciertamente urgente, pero debe realizarse atendiendo -en la medida de lo posible- a los efectos colaterales adversos que esta transformación genera en los derechos fundamentales que el Derecho reconoce y resguarda.

Un principio ambiental clave que sustenta e impulsa esta transformación económica, en mi opinión, de visible influencia preventiva, es el conocido como "contaminador pagador", que preferimos denominar como "internalización de costos ambientales", el cual, con base en la teoría económica, postula que quien crea el riesgo ambiental debe incorporar en su contabilidad los costos que irrogarán las medidas de prevención, mitigación y control de este riesgo.

Lo contrario, es decir, que no sean los causantes del riesgo ambiental sino los afectados por su realización concreta, real o inminente, los que asuman los costos económicos, supondría la aparición de fallas y distorsiones en los mercados tanto de bienes como de servicios.

Otro lineamiento ambiental con un importante componente económico es el conocido como Responsabilidad Extendida del Productor -en adelante, "REP"-, presente en nuestra legislación sobre materiales y gestión integral de residuos sólidos.

El REP postula que los productores, que dependiendo de la legislación pueden ser los fabricantes, importadores, distribuidores y/o comercializadores, de bienes ambientalmente peligrosos o de uso muy extendido, deben intervenir económicamente en la gestión posconsumo de los bienes que fabricaron o comercializaron. Asimismo, con sustento en este "principio" se exige a los productores obligaciones relacionadas con el análisis ambiental del ciclo de vida de sus productos, desde el diseño hasta la fase de gestión post consumo, con la finalidad de promover el ecodiseño y la valorización de los residuos sólidos. 
La incorporación de la gestión de riesgos ambientales en los precios deberá ser gradual para evitar efectos recesivos exagerados que terminen por producir efectos sociales contrarios a la mejora ambiental, de ahí la importancia del principio de gradualidad y no reversibilidad ambiental para lograr una transición hacia una economía diferente que contabilice la escasez del ecosistema y que podrá recibir el nombre de economía verde, carbono neutral, circular, etc.

La tecnología desempeña un rol fundamental reduciendo los costos para acceder a soluciones más eficaces para mitigar la polución o crear alternativas a sustancias o bienes cuyo desempeño implica altos impactos ambientales. En este orden de ideas, los costos de la tecnología forman parte de las ecuaciones que utilice el Derecho Ambiental para enfrentar los problemas que se propone resolver.

\section{Sustrato metajurídico.}

Aceves, citando a Jaquenod, reconoce en el Derecho Ambiental otra característica particular, siendo esta su sustrato técnico metajurídico. Así: "Sustrato técnico metajurídico. - La normativa ambiental requiere un basamento, además de ético y social, también rigurosa y estrictamente técnico, toda vez que se basa en circunstancias científicas" .

La ciencia y la tecnología constituyen las ventanas a través de las cuales vemos y entendemos las dinámicas ecosistémicas, revelan causalidades, interrelaciones e interacciones, nos alertan de peligros y nos ofrecen soluciones, su metalenguaje pugna por ingresar a las normas ambientales transformando sus contenidos e imponiendo retos a los abogados ambientalistas.

El estado del ambiente muchas veces se encuentra determinado por la presión que ejercen las actividades humanas sobre él. Siguiendo con el ejemplo sobre la calidad del aire urbano, este se encuentra de alguna manera influencia- do por las emisiones producidas por el hombre con ocasión de actividades como el transporte, la producción de energía eléctrica, actividades industriales o la agricultura, entre otras.

La emisión de gases como el monóxido de carbono, dióxido de carbono, partículas finas, tales como el material particulado de diversos tamaños, óxidos de nitrógeno, compuestos orgánicos volátiles, entre muchísimos otros contaminantes, terminan integrándose y reaccionado con el aire que respiramos, el cual es monitoreado a través de estaciones que arrojan datos sobre la concentración de estos y otros contaminantes. Los datos por lo general se expresan en microgramos por metro cúbico y representan el promedio en un lapso de tiempo, por ejemplo:

Material particulado menor a 10 micras (PM10): 77 microgramos/metro cúbico, promedio en 24 horas.

Esta información representa solo un dato entre miles que pueden ser necesarios para caracterizar la mezcla de gases, partículas y aerosoles que componen el aire respirable solo en un momento determinado, ya que como sospecharán la composición del aire es extremadamente cambiante, debido a que es un fluido.

Cada contaminante del aire interactúa de diversa forma con los elementos vivos y no vivos del entorno, por lo tanto, la contaminación es siempre un concepto relativo. La relatividad de la contaminación está en relación a lo que se decide proteger; por ejemplo: si el riesgo que deseo gestionar son las enfermedades respiratorias agudas en población vulnerable — niños menores de 5 años, pacientes con enfermedades respiratorias crónicas y adultos mayores- los índices de exposición y tolerancia a ciertos contaminantes serán menores a lo que podrían ser aceptables para una población adulta y sana. En este orden de ideas, lo que para un niño con

7. Carla Aceves, «Nacimiento y Ubicación Jurídica de la Disciplina del Derecho Ambiental», en Bases Fundamentales de Derecho Ambiental Mexicano (Ciudad de México: Editorial Porrúa, 2004). 
asma sería un aire respirable peligroso, para un adulto sano no lo será en igual magnitud.

Otro ejemplo de lo dramáticamente relativo que podría ser el concepto de "contaminación" o riesgo lo ofrece la investigación del Doctor Gino Bianchi Mosquera, la Doctora Linda Hall, el Ingeniero John Connor y la Geóloga Lila Berckley, quienes postulan una metodología para desarrollar niveles de protección ecológicos específicos para un sitio determinado y concretamente para el componente suelo, los cuales son decenas de veces menos conservadores que los Estándares de Calidad Ambiental para suelos - ECA-S- ${ }^{8}$.

Se trata, en pocas palabras de reemplazar los ECA-S como parámetro de referencia, debido a que se basan en la protección de la salud humana y en su lugar construir nuevos valores de referencia, los cuales serían basados en determinadas especies de fauna que se encuentran en el área de influencia del proyecto y que deseamos proteger.

En ese sentido, Bianchi et al. sostienen que: "Según la sustancia química y el sitio, los criterios basados en salud humana pueden resultar inadecuados para la protección del ecosistema, dado que estos criterios pueden ser ya sea insuficientemente protectores o bien excesivamente protectores de la especie ecológica pertinente" ${ }^{9}$.

Las normas ambientales incorporan información proveniente de la ciencia y tecnología, de ahí la fisonomía técnica de ellas, sin embargo, el componente técnico no determina que una norma sea ambiental, en mi opinión la esencia ambiental de la regulación proviene de su propósito, los principios ambientales a los que responde, su concepción sistémica, etc.
El Derecho al momento de regular simplifica, la ciencia cuando investiga amplifica; cada descubrimiento implica nuevos cuestionamientos, por ello si bien la tecnología y la ciencia forman parte del Derecho Ambiental, su integración no es automática, su inclusión debe atravesar un proceso de pertinencia regulatoria que considera además de lo señalado en el párrafo anterior, su compatibilidad con el sistema jurídico.

\section{Multidisciplinario.}

Siguiendo a la misma autora y en estrecha vinculación con la característica que Aceves denomina como "sustrato técnico metajurídico", el Derecho Ambiental es multidisciplinario:

"El derecho ambiental debe abarcar de modo integral, las diferentes interrelaciones que interactúan en la realidad y que dan origen al carácter sistémico de esta rama del derecho (...) Se deben combinar de manera interdisciplinaria las colaboraciones de especialistas técnicos (biólogos, químico, físicos, geólogos, economistas, etc.) a fin de delimitar una realidad objetiva, y tomar las medidas jurídicamente adecuadas para alcanzar los objetivos de esta rama jurídica" ${ }^{10}$.

Esta característica hace del Derecho Ambiental uno en el que la regulación evoluciona, se interpreta utilizando como insumos información producida en las diferentes ramas del saber humano, lo que implica el trabajo colaborativo de juristas, científicos sociales y naturales y tecnólogos.

Las reglas que componen el Derecho Ambiental se forjan en el crisol de la multidisciplinariedad debido a que el objeto de esta rama jurídica es el todo; todo que nos incluye, rodea y

8. Mediante Decreto Supremo N 011-2017-MINAM se aprobaron los Estándares de Calidad Ambiental-ECA Suelo y, mediante Decreto Supremo N 012-2017-MINAM, se aprobaron los Criterios para la Gestión de Sitios Contaminados. Ambos dispositivos legales fueron publicados en el Diario Oficial El Peruano el 2 de diciembre de 2017.

9. Gino Bianchi et al., «Un enfoque práctico para el desarrollo de niveles de evaluación ecológicos para suelo en áreas de influencia de operaciones mineras andinas», Minería 484 (2018): 73-78.

10. Carla Aceves, Op. Cit., 75. 
trasciende, el todo entendido como interrelación de elementos que sustentan la vida en todas sus formas.

El saber humano se encuentra compartimentado en ramas de la ciencia y todas ellas tienen como objeto de estudio alguna parte de ese todo que denominamos ambiente.

El Derecho Ambiental es el lugar donde se encuentran las ciencias con los juristas con la finalidad de crear las reglas que regulen las conductas humanas que son necesarias evitar o impulsar en bien del funcionamiento del todo. El Derecho Ambiental desde esta perspectiva es un Derecho de síntesis, síntesis resultante de las confrontaciones dialécticas de científicos, ideólogos, políticos, tecnócratas, masas, activistas y juristas.

\section{Vinculado a resultados — riesgos, crisis-}

El Derecho Ambiental surge de una crisis y esta génesis le confiere un propósito primigenio: resolverla. Desde sus inicios el Derecho Ambiental se confronta con la resolución de un problema, por ello su evolución se encuentra determina en gran medida por la forma en que el problema es primero percibido, concebido, estudiado y luego abordado para solucionarlo.

El surgimiento del Derecho Ambiental determina que intuitivamente se espere de él un resultado concreto y muchas veces hasta mensurable, de ahí su cercanía con las políticas públicas relacionadas con los temas ambientales, vale decir, casi todas de una u otra forma.

De la cercanía del Derecho Ambiental con la política pública se desprende, desde mi opinión, la estrategia del riesgo ambiental. Para lograr sus resultados el derecho ambiental prioriza en su actuar la gestión de riesgos ambientales como su primera prioridad estratégica, lo que está en línea de coherencia con los principios preventivo y precautorio.

La necesidad de lograr resultados mensurables, en términos por ejemplo de calidad ambiental, plantea la necesidad de definir referencias con- tra las cuales comparar la situación ambiental, los avances o retrocesos. Normalmente la referencia más utilizada en las legislaciones ambientales - cuando tratamos temas de calidad ambiental - ha sido la salud humana, sin embargo, las referencias pueden basarse en especies receptoras determinadas, como lo plantea el trabajo de Gino Bianchi, u otras situaciones cuyo foco sea más directamente el ambiente y menos el ser humano.

Esta característica acerca al Derecho Ambiental a la lógica que tienen las políticas públicas, se integran en un marco conceptual los objetivos, las estrategias, las medidas e instrumentos lo que explica la gran dinámica o índice de variabilidad de sus normas. Así, las normas ambientales, a diferencia de otros tipos de normas jurídicas, están sujetas a la medición de sus resultados y a su calibración permanente, más allá de su natural evolución derivada del principio de gradualidad y no reversibilidad.

La sistémica nos ayuda también a comprender qué significa que el Derecho Ambiental es una rama jurídica con resultado. En efecto, si seguimos una aproximación sistémica a la pregunta podemos aceptar la aparente paradoja que cobija la respuesta, el Derecho Ambiental persigue en simultaneo un resultado general y muchísimos resultados específicos. El ambiente incluye procesos ecosistémicos globales y microprocesos, todos interconectados e interdependientes, de los cuales depende la enorme diversidad de la vida con la que estamos conectados.

El Derecho Ambiental será enjuiciado constantemente por su eficacia y eficiencia en conseguir los resultados de mejora ambiental que prometen sus instrumentos, sin embargo, los resultados serán juzgados utilizando las referencias que la ciencia y la tecnología postulen como las apropiadas, sin que exista derecho de apelación por parte de otro operador no científico del saber humano.

\section{CIERRE DE LA PRIMERA PARTE}

El Derecho Ambiental positivo, sospecho - no se ha medido, que yo sepa-, tiene una tasa

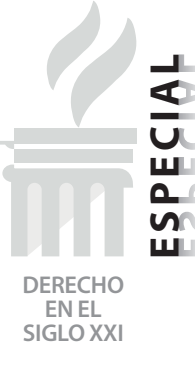


de cambio y evolución más dinámica que otras ramas del Derecho, esta situación se explica en parte por las características que hemos reseñado brevemente hasta aquí: sistémica, preventiva y precautoria, metajurídica, multidisciplinaria, renovador en lo económico y vinculado a resultados.

El Derecho Ambiental no tiene más de 50 años, es una rama joven del Derecho y por ello su identidad, contornos y fronteras son aún borrosos, no obstante, en este medio siglo podemos identificar lo que podemos denominar proto-características del Derecho Ambiental, las cuales deben de terminar de definirse y consolidarse, luego de atravesar un proceso de cambio vertiginoso propio de todo comienzo.

El equilibrio que quizás llegará a tener en un futuro el Derecho Ambiental será, como es usual, el resultado del equilibrio entre dos fuerzas antagónicas: por un lado las fuerzas del cambio, que se anclan en los descubrimientos y alertas cada vez más urgentes provenientes de la comunidad científica y tecnológica, la sociedad civil en general y la sociedad civil organizada en torno a la preocupación ambiental; y por otro lado, las fuerzas de la conservación, las cuales se muestran escépticas respecto a los escenarios futuros descritos por la ciencia y/o propugnan una adecuación progresiva de los sistemas económicos a las posibilidades del ambiente. En esta confrontación de posiciones el jurista ambiental juega un rol fundamental, al resguardar la integridad de la sistemática jurídica ambiental. Seremos defensores de la simplificación que domina a la ciencia jurídica en una trinchera -Derecho Ambiental-asediada por la complejidad.

En la parte siguiente quisiera relevar, en base a dos casos prácticos, la forma concreta en que se relacionan la ciencia, la tecnología y el Derecho Ambiental. El primero es el problema ambiental que conocemos como agujero en la capa de ozono y el segundo, a propósito de la propuesta metodológica del Doctor Gino Bianchi y otros investigadores.

\section{CIENCIA, TECNOLOGÍA Y RIESGOS AM- BIENTALES}

\section{Caso 1.}

Carl Sagan, en su libro "Un Punto Azul Pálido, una visión del futuro humano en el espacio" explica como la investigación científica relativa a constituyentes menores de la atmósfera del planeta Venus terminó por detectar el peligro que supone la pérdida de la capa de ozono de nuestro planeta.

Como hoy sabemos las moléculas de clorofluorocarburos - CFC - una vez que ascienden hasta la estratosfera son descompuestas por efecto de la luz ultravioleta proveniente del Sol, y las moléculas de clorina así liberadas destruyen las moléculas de ozono, dejando a la Tierra sin su protección natural contra la luz ultravioleta, radiación que es causante de innumerables problemas para la vida en su conjunto.

La alerta sobre este problema ambiental global se la debemos a Sherwood Rowland y Mario Molina, científicos de la Universidad de California, como lo recuerda Sagan:

"En sus cálculos originales, Rowland y Molina emplearon constantes tipos de las reacciones químicas en las que participa la clorina y otros halógenos, que habían sido medidas en parte con apoyo de la NASA. ¿Por qué la NASA? Porque la atmosfera de Venus contiene moléculas de clorina y fluorina, y los aerónomos planetarios se proponían investigar lo que está sucediendo allin'1.

Sagan también afirma que existe unanimidad en la comunidad científica planetaria respecto del efecto que sufren las moléculas orgánicas

11. Carl Sagan, Un punto azul pálido: Una visión del futuro humano en el espacio (Barcelona: Editorial Planeta S.A., 2006), 222. 
ante la carencia de una capa de ozono, esto debido a la investigación que realiza la comunidad científica en el planeta Marte. Así: "La luz ultravioleta del Sol alcanza la superficie de Marte sin topar con el obstáculo de una capa de ozono; si hubiera algo de materia orgánica, sería rápidamente aniquilada por la luz ultravioleta en sí y por sus producto de oxidación"122.

Producida la alarma relativa al inminente peligro que suponía la amplificación del agujero en la capa de ozono, 197 países ratificaron la Convención de Viena para la protección de la capa de ozono, que entró en vigencia en 1987, dos años después, en enero de 1989, inició la vigencia del Protocolo de Montreal Relativo a las Sustancias que Agotan la Capa de Ozono -en adelante, el "Protocolo de Montreal"-, el cual contiene medidas concretas para el control y reducción de la producción y demanda de las sustancias que destruyen la capa de ozono.

En nuestro país, el Congreso ratificó la Convención de Viena en noviembre de $1988^{13}$ y el Protocolo de Montreal en marzo de $1993^{14}$, luego, es a partir del año 2000 que el hoy denominado Ministerio de la Producción aprueba las normas para la aplicación efectiva del Protocolo de Montreal, a través del Decreto Supremo $N^{\circ} 033-2000-$ ITINCl y demás normas modificatorias ${ }^{15}$.

En este caso la ciencia identificó las causas - liberación en la atmosfera de ciertos compuestos claramente identificados-, el problema
- la pérdida de la capa de ozono y el aumento de la intensidad de radiación ultravioleta consecuente- - y sus efectos - diversos tipos de cáncer y efectos destructivos para la vida en general-. La ciencia también nos ofreció datos sobre el potencial destructor de ozono de cada tipo de compuesto ${ }^{16}$.

Por su parte, la tecnología aportó moléculas sustitutas para reemplazar las sustancias agotadoras de la capa de ozono - en adelante, "SAO"-, sin embargo, algunos sustitutos seguían causando el mismo problema, pero con factores de destrucción del ozono muy por debajo del que tenía la sustancia que reemplazaban.

La solución implicó un acuerdo mundial muy extendido, de prácticamente todos los países del mundo, para limitar la producción y el uso de las $\mathrm{SAO}$, dentro de los plazos que cada país podía establecer, según sus particularidades.

¿Dónde encontramos al Derecho Ambiental en este caso? El Derecho Ambiental no lo encontramos en el derecho positivo; las normas para implementar el Protocolo Montreal fueron aprobadas por el Ministerio de la Producción, los textos de los dispositivos legales hacen referencia a sustancias químicas difíciles de pronunciar, plazos y restricciones a la importación, producción y venta de estas sustancias, sin embargo, el Derecho Ambiental está muy presente.

Todas las restricciones sobre el comercio que impone el Protocolo de Montreal se fundan en

12. Ibíd., 223.

13. El Convenio de Viena para la protección de la capa de ozono de ozono fue ratificado por Resolución Legislativa Nº 24931 del 29 de noviembre de 1988.

14. El Protocolo de Montreal relativo a las sustancias que agotan la capa de ozono fue ratificado por Resolución Legislativa $N^{\circ} 26178$ del 29 de marzo de 1993.

15. Al respecto, la normativa mencionada comprende:

a) Resolución Ministerial N²77-2001-ITINCI/DM, se aprueban los alcances del Decreto Supremo No 033-2000-ITINCI, publicado el 30 de noviembre de 2001; y

b) Decreto Supremo Nº03-2015-PRODUCE, modifican el Decreto Supremo Nº33-2000-ITINCI, publicado el 4 de febrero de 2015.

16. Cfr.: Protocolo de Montreal: anexo A, anexo B, anexo C y anexo E. 
la necesidad de solucionar un problema ambiental, con potenciales implicancias negativas sistémicas para la vida.

No interesa tanto qué diga el texto de la norma legal o si ésta versa sobre materia, tributaria, penal, civil o administrativa, la esencia ambiental la encontramos en cómo estas normas fueron concebidas, cómo se interrelacionan -enfoque sistémico-, qué propósito persiguen y cómo debemos enfrentar su exégesis.

Evidentemente la acción emprendida para enfrentar el problema de la capa de ozono fue sistémica en muchos sentidos, tuvo las características de ser una acción multilateral, simultánea y organizada en todo el mundo, la cual involucró a países, industrias, comercio y ciudadanía en múltiples formas, considerando acciones de mitigación y adaptación, y poniendo en acto una muy extensa gama de instrumentos de gestión, planificación, participación, etc.

Las investigaciones científicas sobre la capa de ozono ciertamente tuvieron fisuras, dudas, y cuestionamientos ${ }^{17}$, sin embargo, ello no fue un motivo para paralizar o retardar la acción necesaria para enfrentar los riesgos en ciernes que, si bien no fueron vistos en alta definición, sí pudieron ser identificados con el grado de suficiencia que exige el principio precautorio, principio que impone un camino de acción firme ambiental incluso en contextos donde la duda podría ser suficiente como para paralizar la acción de otras ramas del Derecho.

El principio precautorio explica y sustenta una mayor sensibilidad o atención del Derecho Ambiental a lo que sucede en la ciencia, nos dice: ante problemas como el cambio climá- tico, la pérdida de biodiversidad o la capa de ozono, seremos menos escépticos frente a la ciencia porque la incredulidad puede comprometer el propósito esencial del Derecho Ambiental.

Otro principio que jugó un rol definitivo fue el de internalización de costos ambientales, el cual justificó todos los impactos económicos derivados de las restricciones impuestas por el Protocolo de Montreal que, sin embargo, fueron moduladas a través de la concesión de plazos para su implementación, los cuales fueron establecidos a la luz del principio de gradualidad y no reversibilidad ambiental.

El Protocolo de Montreal es considerado como unos de los más exitosos de la humanidad para resolver un problema ambiental. La medición de sus resultados apunta a que la capa de ozono volverá al estado previo al 1980 antes del año 2050.18

\section{Caso 2.}

Otro caso a partir del cual podemos analizar la interacción entre ciencia, tecnología y Derecho Ambiental es el que presentamos en la sección relativa al carácter metajurídico del Derecho Ambiental, donde presentamos una sinopsis de la metodología del Doctor Gino Bianchi y otros investigadores, para construir parámetros alternativos a los ECA-S, los cuales se enfocarían en proteger específicamente las especies objetivo de la evaluación, de acuerdo con sus particulares factores de exposición y valores de toxicidad de referencia. En caso no se encontraran disponibles estos factores para determinadas especies se podrían usar, sostienen los investigadores, especies equivalentes, como se muestra en la tabla ${ }^{19}$ siguiente:

17. Uno de los cuestionamientos fue el de señalar como poco probable que las moléculas de los CFC pudieran ascender hasta la estratosfera cuando son más pesadas que el aire.

18. David Fahey, «Veinte Preguntas y Respuestas sobre la Capa de Ozono», 2002, 37, https://www.gob.mx/cms/ uploads/attachment/file/31180/20PreguntasdeOzono.pdf.

19. Gino Bianchi et al., Op. Cit., 73-78. 
Tabla 1. Gremio Ecológico de las Especies Andinas y Equivalentes

\begin{tabular}{c|c|c}
$\begin{array}{c}\text { Especie andina } \\
\text { (género, especie) }\end{array}$ & $\begin{array}{c}\text { Especie equivalente } \\
\text { (género, especie) }\end{array}$ & $\begin{array}{c}\text { Gremio ecológico, especies } \\
\text { andinas y equivalentes }\end{array}$ \\
$\begin{array}{c}\text { Cóndor andino } \\
\text { (Vultur gryphus) }\end{array}$ & $\begin{array}{c}\text { Gavilán colirrojo } \\
\text { (Buteo jamaicensis) }\end{array}$ & Aviar carnívoro \\
\hline $\begin{array}{c}\text { Gato andino } \\
\text { (Leopardus jacobitus) }\end{array}$ & $\begin{array}{c}\text { Gato montés } \\
\text { (Lynx rufus) }\end{array}$ & Mamífero carnívoro \\
\hline $\begin{array}{c}\text { Churrete ventriblanco } \\
\text { (Cinclodes atacamensis) }\end{array}$ & $\begin{array}{c}\text { Chorlo gritón } \\
\text { (Charadrius vociferus) }\end{array}$ & Mamífero herbívororo \\
\hline $\begin{array}{c}\text { Vicuña } \\
\text { (Vicugna vicugna) }\end{array}$ & $\begin{array}{c}\text { Ciervo mulo } \\
\text { (Odocoileus hemionus) }\end{array}$ & Mamífero herbívoro \\
\hline $\begin{array}{c}\text { Vizcacha } \\
\text { (Lagidium peruanum) }\end{array}$ & $\begin{array}{c}\text { Perrito de la pradera de cola } \\
\text { negra (Cynomys ludovicianus) }\end{array}$ & Aviar granívoro \\
\hline $\begin{array}{c}\text { Jilguero cara gris } \\
\text { (Sicalis uropygialis) }\end{array}$ & $\begin{array}{c}\text { Faisán común } \\
\text { (Phasianus colchicus) }\end{array}$ & \\
\hline
\end{tabular}

La citada investigación científica, además de ser interesante, es en muchos sentidos retadora para el Derecho Ambiental, debido a que plantea que, si lo que se pretende evaluar es el impacto de un proyecto en un sitio determinado a un conjunto de especies de fauna silvestre también determinado, quizás los ECA-S no sería el mejor parámetro de referencia por su generalidad.

Lo propio podemos afirmar del ECA de agua, el cual al ser un referente general evidentemente no se adapta a las particularidades ecosistémicas de todos los cuerpos de agua presentes en un país.

En términos de calidad ambiental, la referencia más relevante que considera la legislación ambiental peruana la constituyen las normas ECA. Nuestra sistemática jurídica ambiental ubica a los ECA como el eje fundamental al cual se integran muchos de los instrumentos de gestión ambiental, tales como los estudios ambientales - detallados, semidetallados y declaración de impacto ambiental, etc.-, la fiscalización ambiental, la participación ciudadana, etc.
La metodología propuesta propone, con base en el sentido común y la ciencia, reemplazar el ECA, que como sabemos se construye con base en salud humana, por referentes especiales construidos para proyectos, ecosistemas y especies específicas.

En tal sentido, podría surgir una norma ambiental que establezca lo siguiente: "En caso de proyectos extractivos sobre los $3500 \mathrm{msnm}$ en cuya área de influencia no existan asentamientos humanos, se podrá suspender la aplicación de los ECA-S como criterio de evaluación de los impactos. Los ECA-S deberán ser reemplazados por un estudio específico que sustente valores de referencia diferentes a los ECA-S, y que sean protectores de las especies de flora y fauna que habitan la zona de influencia del proyecto."

Esta norma ficticia cuenta con un claro sustento científico, sin embargo, su inclusión en la regulación ambiental dependerá del análisis de su compatibilidad con los principios del Derecho Ambiental y la sistemática jurídica ambiental, tarea que corresponde desempeñar a los juristas ambientales. 
¿Qué implicancias para el Derecho Ambiental traerá la inclusión de una norma como la esbozada arriba? ¿Corresponde atenuar la simplificación que implica usar los ECA introduciendo valores de referencia especiales para cada proyecto? ¿Favorece esta metodología la participación ciudadana, la mejora? ¿Cuáles serían los efectos sobre la fiscalización ambiental? ¿Es compatible esta norma con los principios ambientales? Son algunas de las preguntas que se harían los operadores especializados del Derecho Ambiental.

\section{A MANERA DE CIERRE}

Se dice que Galileo aconsejaba encontrar las respuestas a los temas sobre Dios en la Biblia y las repuestas a los misterios de la naturaleza en la naturaleza. Al parecer Galileo resentía que los hombres de su tiempo buscaran las respuestas a los fenómenos naturales en la religión.

Hoy las ciencias son, quizás solo por un tiempo más, nuestra ventana a la naturaleza, son ellas las únicas que nos ilustran sobre el ambiente y sus perspectivas, ostentan el monopolio de la información ambiental; por su parte, la tecnología seguirá siendo a la vez problema, solución y esperanza, todo dependerá de la dirección que le marquemos.

El Derecho Ambiental es Derecho, es decir un instrumento que propugna la paz social en base a la imposición de soluciones generales, idealmente concertadas, a problemas que han sido abstraídos y simplificados en supuestos de hecho positivizados.

El Derecho Ambiental encuentra en la ciencia y la tecnología varias cosas: en primer lugar, un método - la sistémica- de conocimiento, regulación, interpretación y comportamiento institucional; su primer mandamiento de actuación, enfocada en el riesgo, preventiva y precautoria; la medición de sus resultados y por lo tanto sus referencias y parámetros; los fundamentos de sus contenidos regulatorios y la fuerza para legitimar las soluciones - imposiciones - a los problemas ambientales. Sin embargo, el Derecho Ambiental como "ciencia social" requiere estar atento a otros factores que de no ser considerados podrían erosionar su eficacia para restringir conductas, uno de ellos es la concepción vigente de bienestar humano, concepto cuya evolución natural se ve acelerada, entre otros factores, por las nuevas tecnologías y que debe ser gradualmente conciliada con las posibilidades reales del ecosistema de sostenerla.

Durante todo el artículo hemos hablado de la tecnología como una herramienta sometida al hombre y de la ciencia como una ventana a la naturaleza, ambos postulados, como todo, cambian. La tecnología, sometida o no, nos viene transformando como especie y sociedad y la ciencia, quién sabe por cuando tiempo más seguirá siendo nuestra ventana confiable a la naturaleza. 